\title{
Trust Execution Environment and Multi-party Computation for Blockchain e-Health Systems
}

\author{
Feriel Yahmed $^{(\bowtie)}$ and Mohamed Abid \\ Unit Hatem Bettaher IRESCOMATH, University of Gabes, Gabes, Tunisia \\ yahmedferiel@gmail.com, mohamed.abid@enig.rnu.tn
}

\begin{abstract}
Blockchain is a rich and attractive domain for researchers since it is independent of "third party" such as Bank or government. This "open" phenomenon does not respect all the security criteria such as private data protection and confidentiality; hence, we cannot trust this approach despite its contributions. Blockchain technology has gained considerable progress in recent years in fields such as e-health. The medical data contains personal and sensitive information that must be preserved. The current Blockchain systems suffer from serious practical limitations, e.g. poor performance, high-energy consumption and lack of confidentiality. On the other hand, Trust Execution Environment TEE is imperfect; it is based on the centralization of data. To avoid data centralization and its limitations, an approach based on collecting the necessary data from distributed database is presented in this paper. Our goals are to protect the user's privacy and to execute it in TEE combined with Multi-party computation MPC. We proof by security analysis that our new solution meets the fundamental criteria of security such as confidentiality and privacy.
\end{abstract}

Keywords: E-health - MPC (Multi-party computation) $\cdot$ BC (Blockchain) TEE (Trust Execution Environment) and Smart Contract (SC) IPFS (Interplanetary File System)

\section{Introduction}

Technological evolution is bringing a profound change to the core of business. Nowadays IT(Information Technology) is not only a productivity tool but also a means of administration and management. It is becoming a strategic and a necessary mean to manage the evolutionary processes of the company's business lines. Therefore, the field of information and communication technologies has become one of the pillars of business.

The information system is an essential element for the company; hence, its innovation must be almost permanent and exploits to the best the new technologies. New network technologies open up new potential for communication and data exchange in different geographical areas. This context has motivated the IT community to take an interest in distributed architectures such as the Blockchain.

Modern systems like Blockchain have become increasingly complex, open, connected and are leading to new challenges. User requirements for security are increasingly demanding. The Blockchain has affected several sectors such as finance, health care, public services, electronic voting, music and the government sector. The reason 
for this enhanced interest is the disappearance of the trusted medium, to operate in a decentralized manner with an acceptable degree of confidence.

The IT community hails the Blockchain as the next great technological innovation. According to Marc Andreessen, co-founder of Netscape and co-writer of Mosaic the Blockchain: "When we sit here in 20 years, we will talk about [Bitcoin and Blockchain technology] the way we talk about the internet today" [1].

\subsection{Blockchain}

The Blockchain is a new technology for storing and transmitting data in a secure and transparent way, it works without a central control body. This technology takes the form of a transaction $\log$ in a peer-to-peer P2P network. These transactions grouped together in the form of blocks, which are linked together. Each block contains data, the hash of the previous block, and a time stamp. Figure 1 represents an example of Blockchain structure.

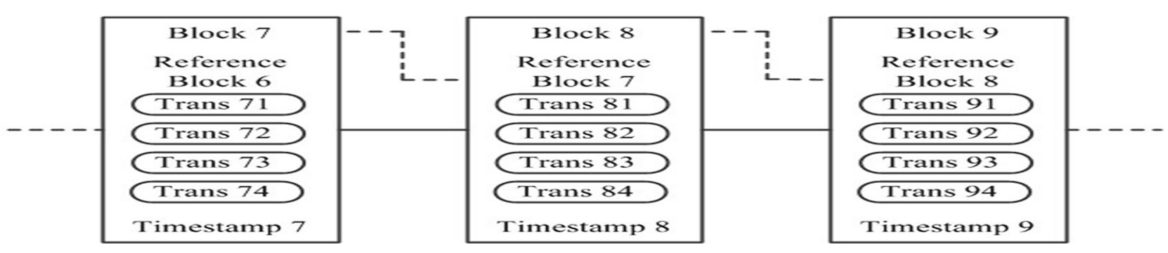

Fig. 1. Blockchain structure [17]

All network nodes back up and verify the data stored in the Blockchain, and consequently provides a strong resilience against attacks that can tamper the integrity of the data.

As this great feature leads to a Blockchain-based implementation of smart contract platforms such as Ethereum [2], several developers have been attracted to build decentralized applications using smart contracts that avoid the need for a central server to manage and maintain the data [3].

The first cryptocurrency based on a Blockchain was Bitcoin in 2008 [7], however the Blockchain has evolved to meet and serve a variety of purposes. The difference between a traditional database and the Blockchain is essentially the storage policy. The Blockchain resides on computer networks However, databases are stored on centralized servers (see Fig. 2). Each one of them has its own advantages and limitations.
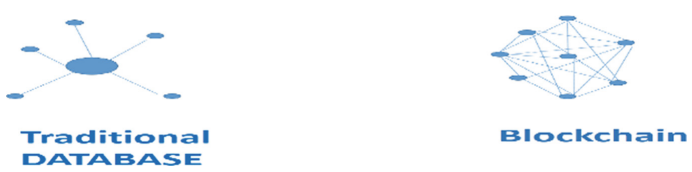

Fig. 2. Traditional DATABASE VS Blockchain 


\subsection{Smart Contract}

Szabo first introduced the term smart contract in 1994, where the smart contract is defined as "a computerized transaction protocol that executes the terms of a contract" [4].

Smart contracts are compiled as byte codes and executed in EVM (Ethereum Virtual Machine) located in miners' computers, which is very similar to Java executed in JVM. When the smart contract operates, it must be packaged by the miner and written into the Blockchain. Each Blockchain in Ethereum has various functions and purposes [5]. Compared with traditional contract, a smart contract is an executable code stored and running in Blockchain. The smart contract may execute independently and automatically without third parties, and these running results are irreversible on Blockchain and are traceable by each participant. The main features of smart contract are given as follows [6]: stability and deterministic features, the same input always produces the same output. Because smart contracts are executable codes stored in Blockchain, every network participant can inspect them. Meanwhile, all the interactions with a smart contract occur via signed messages on the Blockchain and thus every participant can verify and trace the contract's operations.

The structure of this paper is as follows. Section 2 presents a state of the art by analyzing the current situation and motivation. Section 3 describes the steps of the new solution and an e-health use-case. We present the security analysis of the proposed solution in Sect. 4. Conclusion is drawn in Sect. 5.

\section{State of the Art}

We have conducted an intensive research to get the state of the art of Blockchain and smart contracts applications. In the following, we present the existing solutions based on the technologies chosen by researchers.

\subsection{Centralized Database}

The researchers in [8] propose a solution for digitizing certificates, in university use case, in order to improve the conditions and make life much easier using the Blockchain and intelligent contracts. Therefore, it will be possible to have a certificate, wherever the student is and whatever the time, with full security since the access to the data will be done only when people are authorized.

It is true that this solution has contributions in terms of time and speed. However, in our opinion, it does not ensure total security since it puts in danger the private data when they are published in the Blockchain. In addition, the weak point of the solution is the centralization since the data are recorded in the database of the university and if it is broken down, nothing can be done.

\subsection{PKI Public Key Infrastructure}

Existing certificate mechanisms do not dynamically ensure the trustworthiness of a certificate, to solve this weakness Ahmed et al. [9] offer the "smart contract assisted 
PKI". This solution manages trust dynamically in a distributed way and provides better trust experience for users. Despite its contribution, this solution neglects the protection of private data.

\subsection{TEE Trust Execution Environment}

TEE (Trust Execution Environment) [16] is a tamper-proof trusted execution processing environment. It runs on a separate kernel and it can be safely updated.

TEE resists all software and physical attacks. It represents a space for storage execution and secure execution.

In this context, Hawk [10], which is the first TEE, is a smart contract system that provides confidentiality by executing contracts off-chain and posting only zeroknowledge proofs on-chain. The zero-knowledge proofs in Hawk incurs very high computational overhead. Additionally, it was designed for a single compute node called the "manager" which must be trusted for privacy.

There are also some technical inefficiencies such as limited block size and transfer cost. In addition, once the data is stored in the Blockchain, it cannot be modified. This poses certain problems such as falsification during the execution of the contract. In some cases, the contract needs data in real time. The most relevant solution is to store the data off-chain and choose another execution platform more secure and more relevant.

To ensure confidentiality and private data protection, Rifi et al. [11] offer a solution that combines two technologies: Trust Execution Environment TEE and Blockchain, from where data is stored and executed in TEE. It is a platform that ensures data integrity; confidentiality and protection, but it is based on the centralization of data storage. In [11], authors concluded that in order to apply Blockchain technology to EHealth, it should be public, and has three main keys: scalability, secure access to medical data, and data privacy. In this article, the researchers prove that the Blockchain does not have good performances in terms of storage. Therefore, they proposed to store the data off-chain using the IPFS (Interplanetary File System). The new Blockchain technology [15] applied in e-Health identifies new ways to share the distributed view of health data and promotes the advancement of precision medicine, improving health and preventing diseases.

\subsection{IPFS}

IPFS [12] (Interplanetary File system) is a decentralized file sharing platform. It identifies files by their contents. To retrieve file locations and connectivity information from the nodes, this system uses Distributed Hash Table (DHT).

As described in [13], the DHT is primarily a distributed key value store. It uses node identifiers and keys. They must have the same length and a distance metric to easily store and retrieve the information. A node tries to find the nodes in its vicinity, when searching a value and a key. To do this; it uses buckets to keep track of the nodes in the network. The spaces are organized so that each node in the network has precise information about its immediate environment. 
Blockchain and IPFS [14] are based on similar concepts of decentralized networks. However, each one of them has its own characteristics. IPFS is a file-sharing system that chops its files. The search for files within IPFS is based on these hashes. Blockchain and IPFS perform very different tasks for their users. It is possible to store files in IPFS while the hashes are stored in Blockchain.

As discussed before, the TTE has many advantages but it is not based on multiparty computation. Therefore, to eliminate the centralization of data, we try to link between IPFS, TEE and the Blockchain to have efficient results that respect the security rules.

The use of TEE allows the user to store his/her data in TEE and to execute his/her smart contract. To access to the latter, the user must enter his/her public hash key; TEE compares it with the list of public hash keys; if it is compatible with a public hash key, he/she can access to it. The Blockchain is used to transfer smart contract from user to TEE (see Fig. 3 Smart contract with TEE). To import data from IPFS, the user should put the IPFS Hashes and a time stamp in the smart contract (see Fig. 3 Smart contract with IPFS). As a conclusion, executing smart contract in Blockchain suffers from many problems such as poor performance, high-energy consumption.

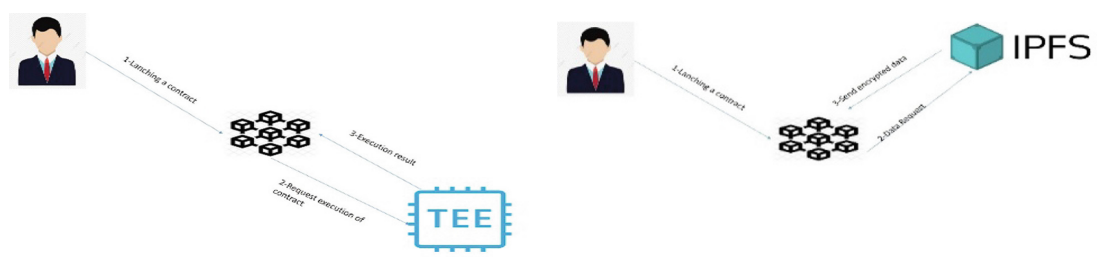

Fig. 3. Smart contract with TEE VS Smart contract with IPFS

\section{The Proposed Solution}

With the technical progress, many technologies are developing, a huge amount of exchanged data will appear, and the exchange of data is carried out from different locations and different sources. In order to ensure all these criteria, we must have a solution that provides: Confidentiality, Authenticity, Integrity, Decentralization and privacy in two phases: Data Storage and Smart contract' execution.

\subsection{Architecture and Security Parameters}

Figure 4 presents the architecture of the new solution which is composed by two phases:

a) Data Storage

In this phase, the user stores hislher encrypted data in different places. To access to it, we need to process some cryptographic steps. 


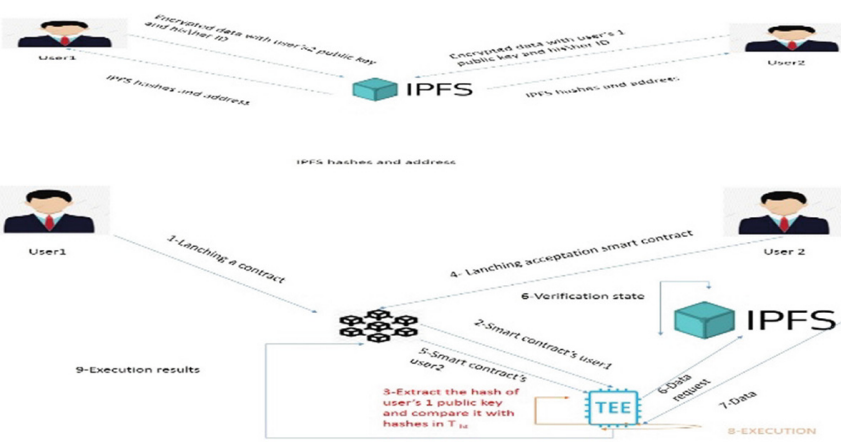

Fig. 4. Proposed architecture

b) Smart contract's execution

Before this operation, we need to transfer the smart contract using Blockchain. After that, the TEE "imposes" some protocols to access to it. Also, TEE controls missing data and imports them from DDB (Distributed Data Base) The approach proposed in Article [18] can be used to collect data either from IPFS or from users. After that, it executes the smart contract and returns the result to the Blockchain.

Table 1 contains all the security parameters of the communicating parties in ehealth scenario.

Table 1. Security parameters

\begin{tabular}{l|l}
\hline Parameters & Description \\
\hline $\mathrm{H}_{\text {IPFS }}$ & Hashes IPFS \\
\hline $\mathrm{H}_{\text {Kpub }}$ & Hashes public key \\
\hline $\mathrm{K}_{\text {pub }}$ & Public key \\
\hline $\mathrm{K}_{\text {priv }}$ & Private key \\
\hline$@_{\text {IPFS }}$ & IPFS address \\
\hline $\mathrm{T}_{\mathrm{ht}}$ & Hashes table in TEE \\
\hline $\mathrm{L}_{\mathrm{DD}}$ & List of demanded data \\
\hline EN & Encrypt with public key \\
\hline
\end{tabular}

\subsection{Steps of the New Solution}

Next, we describe, step by step, the exchanges in the new solution:

a) User's subscription (Data Storage)

A user accesses an IPFS and stores his/her encrypted message with homomorphic cryptography. The encryption ensures data security since only authorized persons have the right to access encrypted data. IPFS sends to the user its @ ${ }_{\text {IPFS }}$ and $\mathrm{H}_{\text {IPFS }}$. 
1 - User 1 sends hisher Id and $\mathrm{EN}_{\mathrm{Kpubuser2}}$ (Data) to the IPFS.

2 - IPFS sends @ ${ }_{\text {IPFS }}$ and $\mathrm{H}_{\text {IPFS }}$ to User2

3 - User 2 sends his ther Id and $\mathrm{EN}_{\text {Kpubuser1 }}$ (Data) to the IPFS.

4 - IPFS sends @ ${ }_{\text {IPFS }}$ and $\mathrm{H}_{\text {IPFS }}$ to User1

b) Smart contract execution

1 - User 1 signs his her smart contract with his/her private key $\mathrm{K}_{\text {priv }}$ (this smart contract demands @ IPFS $_{\text {and }} \mathrm{H}_{\text {IPFS }}$ from the acceptation smart contract) and sends it to the Blockchain.

2 - The Blockchain sends the signed Smart Contract to the TEE

3 - TEE extracts the hash $\mathrm{H}_{\mathrm{kpub}}$ and compares it with the hashes in $\mathrm{T}_{\mathrm{ht}}$

4 - User 2 signs his her contract that contains his Vher $\mathrm{K}_{\text {priv }}$ and sends it to the Blockchain.

5 - The Blockchain sends the signed Smart contract to the TEE. The latter extracts the $\mathrm{H}_{\mathrm{Kpub}}$ of User 2 and compares it with the hashes in $\mathrm{T}_{\mathrm{h}}$. After that, it builds a file that contain missing data.

6 - TEE sends the file that contains the $\mathrm{L}_{\mathrm{DD}}$ (List of demanded data), @ ${ }_{\mathrm{IPFS}}$, $\mathrm{H}_{\text {IPFS }}$ and timestamp (from acceptation smart contract) to IPFS

7 - IPFS verifies the TEE request that contains @ ${ }_{\text {IPFS }}, H_{\text {IPFS }}, L_{\text {DD }}$ and timestamp after that it sends missing data to the TEE.

8 - TEE executes the smart contract

9 - TEE sends the executed results to the Blockchain.

\subsection{E-Health Use Case}

We present in the following the steps of our solution when applied to e-health scenario. In this use case, we have two actors, the doctor and the patient. Figure 5 shows the steps of this use case.

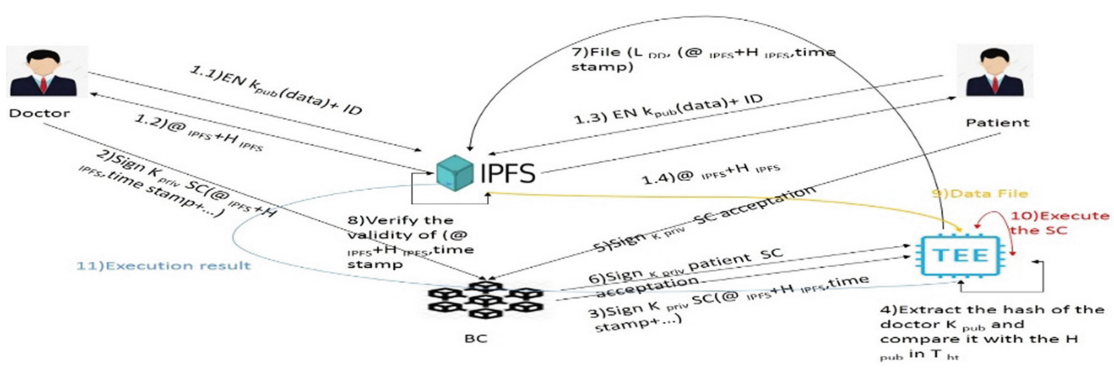

Fig. 5. All the exchanges in e-health scenario

1.1, 1.2, 1.3 and 1.4: Both doctor and patient send $\mathrm{EN}_{\mathrm{Kpub}}$ (data) + ID and receive $@_{\text {IPFS }}+\mathrm{H}_{\mathrm{IPFS}}$

2 - The doctor sends his/her ID and signed smart contract to the Blockchain.

3 - The Blockchain sends this smart contract to TEE 
4 - The TEE receives the smart contract, after it verifies the state of the doctor. Then, it extracts the public key of the doctor and calculates its hash and compares it with the hashes in $\mathrm{T}_{\mathrm{ht}}$

5 - The patient signs smart contract acceptation with hislher private key and sends it to the TEE using Blockchain.

6 - The Blockchain sends the signed SC patient with $\mathrm{K}_{\text {priv }}$ to the TEE.

7 - TEE creates a file that contains a list of demanded data with IPFS hashes, @IPFS and the time stamp and send it to the IPFS.

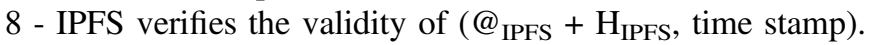

9 and 10 - TEE executes the SC and returns the execution result to the Blockchain.

\section{Security Analysis}

Table 2 presents a comparison between three previous solutions and our proposed solution. We observe that the others solution does not respect all the criteria of security.

When using this approach, the 5 key criteria of security are guaranteed or maintained. in short, user data can only be modified, accessed or deleted by authorized persons (integrity; privacy and integrity).

The IPFS contains the data in a homomorphic way (decentralization) and the execution in TEE will be based on the encrypted data, without forgetting that the TEE and IPFS platforms only allow access to authorized persons.

Table 2. Comparison approaches

\begin{tabular}{|c|c|c|c|c|c|}
\hline Articles & Confidentiality & Privacy & Authenticity & Integrity & Decentralization \\
\hline Hawk [10] & + & + & + & + & - \\
\hline $\begin{array}{l}\mathrm{BC} \text { and } \mathrm{SC} \text { for digital } \\
\text { certificate }[8]\end{array}$ & + & - & + & + & + \\
\hline $\begin{array}{l}\text { Towards using } \\
\text { BC technology for } \\
\text { e-Health data access } \\
\text { management [11] }\end{array}$ & + & - & + & + & + \\
\hline Our solution & + & + & + & + & + \\
\hline
\end{tabular}

\section{Conclusion}

The Blockchain technology has reached a great boom in many sectors such as e-health, e-commerce; e-vote... The main important Blockchain [11] characteristics are: Transparency, no need for third parties and instant access to data since it is replicated on all nodes. To create a secure smart contract based on Blockchain, a new solution is presented which combines three technologies: Blockchain, TEE, and IPFS in order to take advantage of their benefits. This approach is based on collecting the necessary data from distributed database while protecting the user's privacy and executing it in TEE. 
This approach meets the various security criteria such as confidentiality, authentication, integrity, decentralization and protection of private data. this approach can be applied in different domains such as e-health to show the strong points of this one in the ehealth domain it is necessary to link it with the Internet of Things domain [19].

As future work, we need to implement the new solution in order to study its feasibility and measure its performance.

\section{References}

1. Kim, H., Laskowski, M.: A perspective on blockchain smart contracts: reducing uncertainty and complexity in value exchange. In: 2017 26th International Conference on Computer Communication and Networks (ICCCN) (2017). https://doi.org/10.1109/iccen.2017. 8038512

2. Wood, G.: Ethereum: a secure decentralized generalized transaction ledger. Ethereum Proj. Yellow Paper 151, 1-32 (2014)

3. McCorry, P., Shahandashti, S.F., Hao, F.: A smart contract for boardroom voting with maximum voter privacy. In: Kiayias, A. (ed.) FC 2017. LNCS, vol. 10322, pp. 357-375. Springer, Cham (2017). https://doi.org/10.1007/978-3-319-70972-7_20

4. Szabo, N.: Smart contracts (1994). http://www.fon.hum.uva.nl/rob/Courses/ InformationInSpeech/CDROM/Literature/LOTwinterschol2006/szabo.best.vwh.net/smart. contracts.html

5. Magazzeni, D., McBurney, P.: Validation and verification of smart contracts: a research agenda. Computer 50, 50-57 (2017)

6. Braghin, C., Cimato, S., Damiani, E., Baronchelli, M.: Designing smart-contract based auctions. In: Yang, C.-N., Peng, S.-L., Jain, L.C. (eds.) SICBS 2018. AISC, vol. 895, pp. 54-64. Springer, Cham (2020). https://doi.org/10.1007/978-3-030-16946-6_5

7. Nakamoto, S.: Bitcoin: a peer-to-peer electronic cash system (2008). https://bitcoin.org/ bitcoin.pdf

8. Cheng, J.-C., Lee, N.-Y., Chi, C., Chen, Y.-H.: Blockchain and smart contract for digital certificate. In: 2018 IEEE International Conference on Applied System Invention (ICASI) (2018)

9. Ahmed, A.S., Aura, T.: Turning trust around: smart contract-assisted public key infrastructure. In: 2018 17th IEEE International Conference on Trust, Security and Privacy in Computing and Communications/12th IEEE International Conference on Big Data Science and Engineering (TrustCom/BigDataSE) (2018)

10. Kosba, A., Miller, A., Shi, E., Wen, Z., Papamanthou, C.: Hawk: the blockchain model of cryptography and privacy-preserving smart contracts. In: IEEE Security and Privacy (S\&P) (2016)

11. Rifi, N., Rachkidi, E., Agoulmine, N., Taher, N.C.: Towards using blockchain technology for eHealth data access management. In: 2017 Fourth International Conference on Advances in Biomedical Engineering (ICABME) (2017)

12. Benet, J.: IPFS - Content addressed, versioned, p2p file system (draft 3) (2014). https://ipfs. io/ipfs/QmR7GSQM93Cx5eAg6a6yRzNde1FQv7uL6X1o4k7zrJa3LX/ipfs.draft3.pdf

13. Maymounkov, P., Mazières, D.: Kademlia: a peer-to-peer information system based on the XOR metric. In: Druschel, P., Kaashoek, F., Rowstron, A. (eds.) IPTPS 2002. LNCS, vol. 2429, pp. 53-65. Springer, Heidelberg (2002). https://doi.org/10.1007/3-540-45748-8_5. http://dl.acm.org/citation.cfm?id=646334.687801 
14. https://www.edureka.co/community/30534/what-is-the-difference-between-ipfs-andethereum

15. Alonso, S.G., Arambarri, J., López-Coronado, M., de la Torre Díez, I.: Proposing new blockchain challenges in eHealth. J. Med. Syst. 43, 64 (2019)

16. https://www.trustonic.com/news/technology/what-is-a-trusted-execution-environment-tee/

17. https://www.researchgate.net/figure/The-composition-ofblockchain_fig2_327277530

18. El Houda, Z.A., Hafid, A.S., Khoukhi, L.: Cochain-SC: an intra- and inter-domain DDoS mitigation scheme based on blockchain using SDN and smart contract. IEEE Access 7, 98893-98907 (2019)

19. Moudoud, H., Cherkaoui, S., Khoukhi, L.: An IoT blockchain architecture using oracles and smart contracts: the use-case of a food supply Chain. In: 2019 IEEE 30th Annual International Symposium on Personal, Indoor and Mobile Radio Communications (PIMRC) (2019)

Open Access This chapter is licensed under the terms of the Creative Commons Attribution 4.0 International License (http://creativecommons.org/licenses/by/4.0/), which permits use, sharing, adaptation, distribution and reproduction in any medium or format, as long as you give appropriate credit to the original author(s) and the source, provide a link to the Creative Commons license and indicate if changes were made.

The images or other third party material in this chapter are included in the chapter's Creative Commons license, unless indicated otherwise in a credit line to the material. If material is not included in the chapter's Creative Commons license and your intended use is not permitted by statutory regulation or exceeds the permitted use, you will need to obtain permission directly from the copyright holder. 